\title{
An Equivalent LMI Representation of Bounded Real Lemma for Continuous-Time Systems
}

\author{
Wei Xie \\ College of Automation Science and Technology, South China University of Technology, \\ Guangzhou 510641, China
}

Correspondence should be addressed to Wei Xie, weixie@scut.edu.cn

Received 17 September 2007; Accepted 10 January 2008

Recommended by Ondrej Dosly

An equivalent linear matrix inequality (LMI) representation of bounded real lemma (BRL) for linear continuous-time systems is introduced. As to LTI system including polytopic-type uncertainties, by using a parameter-dependent Lyapunov function, there are several LMIs-based formulations for the analysis and synthesis of Hoo performance. All of these representations only provide us with different sufficient conditions. Compared with previous methods, this new representation proposed here provides us the possibility to obtain better results. Finally, some numerical examples are illustrated to show the effectiveness of proposed method.

Copyright (C) 2008 Wei Xie. This is an open access article distributed under the Creative Commons Attribution License, which permits unrestricted use, distribution, and reproduction in any medium, provided the original work is properly cited.

\section{Introduction}

In the past two decades, Ho theory is one of the most sophisticated frameworks for robust control system design. Based on bounded real lemma (BRL), Hœ norm computation problem can be transferred into a standard linear matrix inequality optimization formulation, which includes the product of the Lyapunov function matrix and system matrices. A number of more or less conservative analysis methods / tests are presented to assess robust stability and performance for linear systems with quadratic Lyapunov-function-based results [1], where a fixed quadratic Lyapunov function is found to prove stability and performance of uncertain systems. Especially in [2], for polytopic-LPV systems, a necessary and sufficient condition for quadratic stability can be formulated in terms of a finite linear matrix inequalities (LMIs) optimization problem. The underlying quadratic Lyapunov functions can be also used to derive bounds on robust performance measures. In [3], LMI based-optimization procedures have been proposed to compute $\mathrm{H} 2$ and $\mathrm{H} \infty$ guaranteed cost for linear systems with polytopic-type uncertainties for both continuous time and discrete-time cases. 
To decrease the conservatism of quadratic Lyapunov-function-based results, parameterdependent Lyapunov functions have been used to assess robust stability and to compute guaranteed performance indices. In [4, 5], LMI sufficient conditions for robust stability and Ho guaranteed cost of linear parameter-dependent systems are based on affine-type and polytopic parameter-dependent Lyapunov functions, respectively; a concept called multiconvexity assures that the robust stability condition of uncertain systems is determined by the stability at each vertex of the uncertainty polytope; however, it also renders the results somewhat conservative. More recently, by using polytopic parameter-dependent Lyapunov functions, some less conservative methods are proposed to assess robust stability of uncertain systems in polytopic domains [6-9]. And by introducing some additional variables, extension to $\mathrm{H} 2$ or $\mathrm{H} \infty$ performance for discrete-time systems can be found in [9]. In the continuous-time system case, Ebihara and Hagiwara presented new dilated LMIs formulation for H2 and D-stability synthesis problem [10]. However, this dilated LMIs formulation cannot be extended to Ho synthesis case. In [11, 12], simple modifications of bounded real lemma are introduced for the analysis and the design of continuous-time system with polytopic-type uncertainty; however, the results still are somewhat conservative. de Oliveira et al. presented some sufficient LMI-based conditions to compute $\mathrm{H} \infty$ guaranteed costs for linear time-invariant systems with polytopic-type uncertainties [13], however, the controller design problem has not been considered yet.

In this paper, first, an equivalent linear matrix inequality representation of BRL for linear continuous-time systems is introduced. By introducing a new matrix variable, the new representation is linear with Lyapunov function matrix and system matrix and does not include any product of them. It provides us with a numerical computation method of Hœ norm of LTI plant. Second, by using parameter-dependent Lyapunov function, this representation can also reduce the conservatism that occurs in the analysis and synthesis problems of linear systems with polytopic-type uncertainties. Thereby, based on this representation, robust state feedback synthesis problem is also solved with less conservatism than other methods from literature. We demonstrated the applicability of the new method on two examples. And our results are compared with the standard quadratically stable BRL formulation [1] and an improved LMI condition [11]. The solution to Ho state feedback control of a satellite system with a polytopic uncertainty is also considered in the second example just as in [11].

\section{Preliminary}

Given the following system:

$$
\begin{aligned}
& \dot{x}(t)=A x(t)+B w(t), \\
& z(t)=C x(t)+D w(t),
\end{aligned}
$$

where $x(t) \in \mathfrak{R}^{n}$ is system state vector, $w(t) \in L_{2}^{q}[0, \infty)$ is exogenous disturbance signal, and $z(t) \in \mathfrak{R}^{m}$ is objective function signal including state combination. The system matrices $(A, B, C, D)$ are constant matrices of appropriate dimensions. For a prescribed scalar $\gamma>0$, we define the performance index by

$$
J(w)=\int_{0}^{\infty}\left(z^{T} z-r^{2} w^{T} w\right) d \tau .
$$


Then, from [1], it follows that $J(w)<0$, for all nonzero $w(t) \in L_{2}^{q}[0, \infty)$, if and only if there exists a symmetric positive-definite matrix $P \in \mathfrak{R}^{n \times n}>0$ to satisfy

$$
\left[\begin{array}{ccc}
A P+P A^{T} & P C^{T} & B \\
C P & -I & D \\
B^{T} & D^{T} & -\gamma^{2} I
\end{array}\right]<0
$$

where the symmetric positive matrix $P$ is usually called as Lyapunov function matrix.

This LMI representation is convenient for us to analysis and synthesis nominal control performance for LTI system, when system matrices $(A, B, C, D)$ do not include any parameter uncertainty. However, in the case of linear systems with uncertainty, it will result in very conservative computation for Ho cost $\gamma$ due to the constant Lyapunov function matrix. When a parameter-dependent Lyapunov function is introduced to reduce conservatism in (2.3), it is easy to compute guaranteed performance indices of Hoo norm. Unfortunately, this representation cannot be extended to synthesis control performance problem for linear systems with polytopic-type uncertainty, even though easy state feedback control problem is considered. Therefore, to derive some new equivalent conditions of (2.3) is an efficient resolution to this difficulty. Just like in [11], some simple modifications of BRL are introduced for the analysis and the design of continuous-time system with polytopic-type uncertainty; however, the results still are somewhat conservative. Here, we propose a new equivalent LMI representation of BRL for linear continuous-time systems.

\section{A new LMI representation of BRL}

First, we propose a new equivalent LMI representation of BRL for linear continuous-time systems. Then, this condition is considered to compute Ho guaranteed cost for linear continuoustime system with polytopic-type uncertainty.

Theorem 3.1. There exists a symmetric positive-definite matrix $P \in \mathfrak{R}^{n \times n}>0$ to satisfy (2.3), if and only if there exists a positive symmetric matrix $P$, a general matrix $Q$ satisfying

$$
\left[\begin{array}{cccc}
A Q+Q^{T} A^{T} & P-Q^{T}+r A Q & Q^{T} C^{T} & B \\
P-Q+r Q^{T} A^{T} & -r\left(Q+Q^{T}\right) & r Q^{T} C^{T} & 0 \\
C Q & r C Q & -I & D \\
B^{T} & 0 & D^{T} & -r^{2} I
\end{array}\right]<0
$$

for a sufficiently small positive scalar $r$.

Proof. When a symmetric positive-definite matrix $P$ satisfying (2.3) exists, we always can find a positive scalar $r>0$ as $r<2 \lambda_{1} / \lambda_{2}$, where

$$
\lambda_{1}=\lambda_{\min }\left(-\left(\begin{array}{ccc}
A P+P A^{T} & P C^{T} & B \\
C P & -I & D \\
B^{T} & D^{T} & -\gamma^{2} I
\end{array}\right)\right), \quad \lambda_{2}=\lambda_{\max }\left(\left(\begin{array}{ccc}
A P A^{T} & A P C^{T} & 0 \\
C P A^{T} & C P C^{T} & 0 \\
0 & 0 & 0
\end{array}\right)\right) .
$$


Then applying Schur complement with respect to (3.1) by choosing $Q=P$, we have

$$
\left(\begin{array}{ccc}
A P+P A^{T} & P C^{T} & B \\
C P & -I & D \\
B^{T} & D^{T} & -\gamma^{2} I
\end{array}\right)+\frac{r}{2}\left(\begin{array}{ccc}
A P A^{T} & A P C^{T} & 0 \\
C P A^{T} & C P C^{T} & 0 \\
0 & 0 & 0
\end{array}\right)<0 .
$$

The scalar $r$ makes (3.3) always satisfy.

When a positive symmetric matrix $P$, a general matrix $Q$, and a positive scalar $r>0$

satisfying (3.1) exist, we multiply (3.1) with $T=\left[\begin{array}{llll}I & A & 0 & 0 \\ 0 & C & I & 0 \\ 0 & 0 & 0 & I\end{array}\right]$ on the left and $T^{T}$ on the right, we can get (2.3) directly.

Remark 3.2. It should be noted that the LMIs of Theorem 3.1 are equivalent with well-known standard BRL. Compared with previous study results, improved LMIs-based conditions have been presented as sufficient conditions of BRL in [11,12], though these conditions can be used to design a robust controller based on parameter-dependent Lyapunov functions, however, the results still are somewhat conservative. In [13], by introducing some extra variables, some sufficient dilated LMIs-based conditions have been presented to compute Hoo guaranteed cost, however, the controller design problem has not been considered yet.

We will consider the case of linear systems with polytopic-type uncertainty. Suppose system matrices $(A(a), B(a), C(a), D(a))$ are not precisely known, but belong to a polytopic uncertainty domain $\partial$ as

$$
\partial=:\left\{(A, B, C, D)(a):(A, B, C, D)(a)=\sum_{i=1}^{N} a_{i}\left(A_{i}, B_{i}, C_{i}, D_{i}\right), a_{i} \geq 0, i=1, \ldots, N, \sum_{i=1}^{N} a_{i}=1\right\} .
$$

Since $a$ is constrained to the unit simplex as $a_{i} \geq 0, \sum_{i=1}^{N} a_{i}=1$, these matrices $(A, B, C, D)(a)$ are affine functions of the uncertain parameter vector $a \in \mathfrak{R}^{N}$ described by the convex combination of the vertex matrices $\left(A_{i}, B_{i}, C_{i}, D_{i}\right), i=1, \ldots, N$.

According to Theorem 3.1, linear system with polytopic-type uncertainty as (3.4) is stable and its $\mathrm{H} \infty$ norm is less than a prescribed value of $\gamma$ as the following lemma.

Lemma 3.3. Given system (3.4), its Hoo norm is less than a prescribed value of $\gamma$, if there exist positive symmetric matrices $P_{i}$, a general matrix $Q$ satisfying

$$
\left[\begin{array}{cccc}
A_{i} Q+Q^{T} A_{i}^{T} & P_{i}-Q^{T}+r A_{i} Q & Q^{T} C_{i}^{T} & B_{i} \\
P_{i}-Q+r Q^{T} A^{T} & -r\left(Q+Q^{T}\right) & r Q^{T} C_{i}^{T} & 0 \\
C_{i} Q & r C_{i} Q & -I & D_{i} \\
B_{i}^{T} & 0 & D_{i}^{T} & -\gamma^{2} I
\end{array}\right]<0,
$$

for a scalar $r>0$. Thereby, robust control performance of uncertain continuous-time systems is guaranteed by a parameter-dependent Lyapunov function, which is constructed as

$$
P(a)=\sum_{i=1}^{N} a_{i} P_{i}
$$


By introducing this parameter-dependent Lyapunov function, Ho guaranteed cost $\gamma$ will be obtained less than quadratic Lyapunov-function-based results, where Lyapunov function matrix is a fixed one.

Remark 3.4. Compared with the representation in [11], where the polytopic-type uncertainty is only considered in the matrices $A, B$ or $A, B, C$, the new representation proposed in this paper assumes that polytopic-type uncertainty varies in all of system matrices $(A, B, C, D)(a) \in \partial$. And it also provides less conservative guaranteed Ho cost evaluations than the method [11], as illustrated by numerical examples. Since matrix $Q$ is assumed to be constant one as to system matrices with polytopic-type uncertainty, Lemma 3.3 is also suitable for control synthesis purpose. Furthermore, the conditions (3.5) above will be used to state-feedback synthesis control problem.

\section{State feedback control}

Lemma 3.3 will be extended to solve the state-feedback control problem for linear continuoustime systems with polytopic-type uncertainty.

Consider the following time-invariant system:

$$
\begin{aligned}
& \dot{x}=A(a) x+B_{1}(a) w+B_{2}(a) u, \\
& z=C(a) x+D_{1}(a) w+D_{2}(a) u,
\end{aligned}
$$

where $x, z$ and $w$ are as in (2.1), and $u \in \mathfrak{R}^{r}$ is the control input.

Assume that the system matrices lie with the following polytope as

$$
\begin{aligned}
& \partial_{1}=:\{(\left(A, B_{1}, B_{2}, C, D_{1}, D_{2}\right)(a):\left(A, B_{1}, B_{2}, C, D_{1}, D_{2}\right)(a) \\
&\left.\quad=\sum_{i=1}^{N} a_{i}\left(A_{i}, B_{1 i}, B_{2 i}, C_{i}, D_{1 i}, D_{2 i}\right), a_{i} \geq 0, i=1, \ldots, N, \sum_{i=1}^{N} a_{i}=1\right\} .
\end{aligned}
$$

The state-feedback control problem is to find, for a prescribed scalar $\gamma>0$, the state-feedback gain $F$ such that the control law of $u=F x$ guarantees an upper bound of $\gamma$ to Ho norm.

Substituting this state-feedback control law into (4.1), the closed-loop system can be obtained as

$$
\begin{aligned}
& \dot{x}=\left(A(a)+B_{2}(a) F\right) x+B_{1}(a) w, \\
& z=\left(C(a)+D_{2}(a) F\right) x+D_{1}(a) w .
\end{aligned}
$$

Then, a state-feedback gain $F$ will be solved according to the following theorem.

Theorem 4.1. Given system (4.3), its Ho norm is less than a prescribed value of $\gamma$ if there exist positive symmetric matrices $P_{i}$, matrices $Q, M$ satisfying

$$
\left[\begin{array}{cccc}
A_{i} Q+Q^{T} A_{i}^{T}+B_{2 i} M+M^{T} B_{2 i}^{T} & P_{i}-Q+r A_{i} Q+r M^{T} B_{2 i}^{T} & Q^{T} C_{i}^{T}+M^{T} D_{2 i}^{T} & B_{1 i} \\
P_{i}-Q+r Q^{T} A^{T}+r B_{2 i} M & -r\left(Q+Q^{T}\right) & r Q^{T} C_{i}^{T}+r M^{T} D_{2 i}^{T} & 0 \\
C_{i} Q+D_{2 i} M & r C_{i} Q+r D_{2 i} M & -I & D_{1 i} \\
B_{1 i}^{T} & 0 & D_{1 i}^{T} & -r^{2} I
\end{array}\right]<0
$$

for a scalar $r>0$. If the existence is affirmative, the state-feedback gain $F$ is given by $F=M Q^{-1}$. 


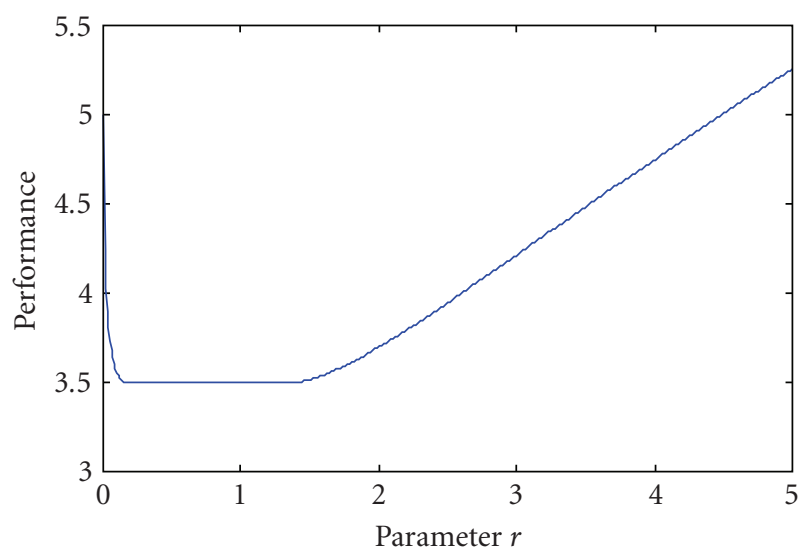

Figure 1: The relation between performance $\gamma$ and $r$.

Remark 4.2. Though some sufficient conditions in [11,12] have been presented to design a robust controller, however, the results still are somewhat conservative. The results of Theorem 4.1 will be compared with the standard BRL formulation and improved LMI conditions [11] with some numerical examples in the next section. It also should be noted, different with Theorem 3.1, as to robust performance analysis and synthesis problems the cost value $\gamma$ will not be a monotonously decreasing function with the decreasing of scalar $r$. In order to obtain the minimum possible $\gamma$, we consider solving (3.5) by iterating over $r$. Although some computation complexity is increased, less conservative results will be obtainable.

\section{Numerical examples}

The approaches developed above are illustrated by some numerical examples; all LMIs-related computations were performed with the LMI toolbox of MATLAB [14].

\subsection{Ho norm computation}

Example 5.1. We consider an uncertain plant [11]:

$$
A(\alpha)=\left[\begin{array}{cc}
0 & 1 \\
-1+\alpha & -1-\alpha
\end{array}\right], \quad B=\left[\begin{array}{l}
0 \\
1
\end{array}\right], \quad C=\left[\begin{array}{ll}
1 & -2
\end{array}\right]
$$

where $\alpha$ is an uncertain parameter that varies in the scope of $|\alpha|<\varsigma$.

It is readily found that the system is stable for $\varsigma=1$, three methods are used to compute Ho guaranteed cost for $\varsigma=0.3777$ as follows:

(1) quadratic Lyapunov-function-based methods [1], Ho guaranteed cost $\gamma=5$;

(2) the method proposed in [11], Ho guaranteed cost $\gamma=4.488$;

(3) the method of Theorem 4.1, $\gamma=3.4963$ for a positive scalar $r$ between 0.15 and 1.43.

The relation between performance $\gamma$ and $r$ is shown as Figure 1. 


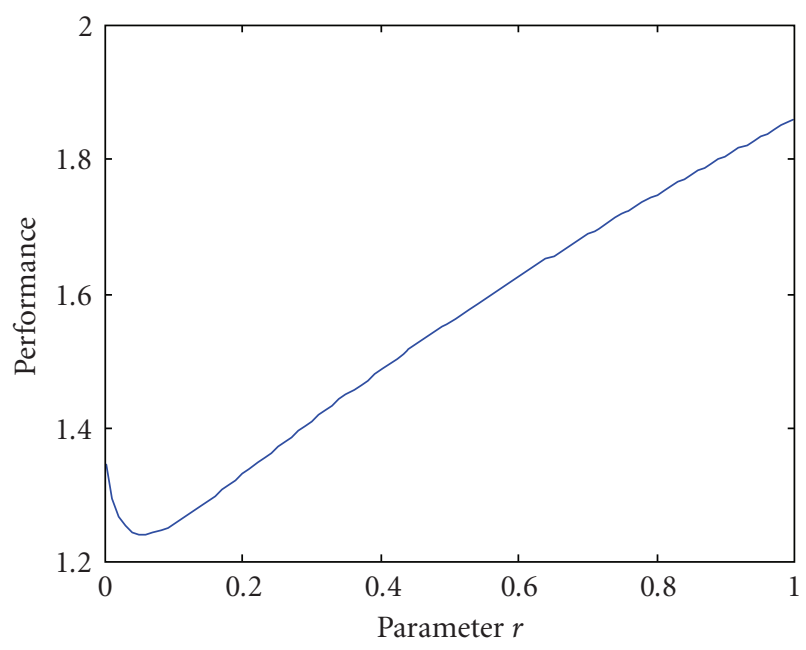

Figure 2: The relation between performance $\gamma$ and $r$.

\subsection{State feedback control}

We consider the problem of controlling the yaw angles of a satellite system that appear in [14]. The satellite system consisting of two rigid bodies joined by a flexible link has the state-space representation as follows:

$$
\begin{aligned}
{\left[\begin{array}{l}
\dot{\theta}_{1} \\
\dot{\theta}_{2} \\
\ddot{\theta}_{1} \\
\ddot{\theta}_{2}
\end{array}\right]=\left[\begin{array}{cccc}
0 & 0 & 1 & 0 \\
0 & 0 & 0 & 1 \\
-k & k & -f & f \\
k & -k & f & -f
\end{array}\right]\left[\begin{array}{l}
\theta_{1} \\
\theta_{2} \\
\dot{\theta}_{1} \\
\dot{\theta}_{2}
\end{array}\right]+\left[\begin{array}{l}
0 \\
0 \\
0 \\
1
\end{array}\right] w+\left[\begin{array}{l}
0 \\
0 \\
1 \\
0
\end{array}\right] u, } \\
z=\left[\begin{array}{llll}
0 & 1 & 0 & 0 \\
0 & 0 & 0 & 0
\end{array}\right]\left[\begin{array}{l}
\theta_{1} \\
\theta_{2} \\
\dot{\theta}_{1} \\
\dot{\theta}_{2}
\end{array}\right]+\left[\begin{array}{c}
0 \\
0.01
\end{array}\right] u,
\end{aligned}
$$

where $k$ and $f$ are torque constant and viscous damping, which vary in the following uncertainty ranges: $k \in\left[\begin{array}{ll}0.09 & 0.4\end{array}\right]$ and $f \in\left[\begin{array}{ll}0.0038 & 0.04\end{array}\right]$.

Just like Example 5.1, three methods are considered to solve this control problem.

(1) With quadratic Lyapunov-function-based methods [1], the minimum guaranteed level of $\gamma=1.557$ can be achieved with $F=-10^{10}$ [0.7391 5.32730 .1337 9.8088].

(2) With the method proposed in [11], the minimum guaranteed level of $\gamma=1.478$ can be achieved for state feedback gain $F=-\left[\begin{array}{llll}579.3 & 4480.6 & 116.2 & 7697.2\end{array}\right]$.

(3) The method of Theorem 4.1, the minimum guaranteed level of $\gamma=1.2416$ can be achieved for $r=0.07$ with state feedback gain $F=-10^{3}\left[\begin{array}{llll}0.1153 & 1.0948 & 0.0307 & 1.5429\end{array}\right]$.

The relation between performance $\gamma$ and $r$ is shown as Figure 2 .

We can find that the cost value $\gamma$ is not a monotonously decreasing function with the decreasing of scalar $r$; Ho guaranteed $\operatorname{cost} \gamma=1.2416$ is obtained for the positive scalar $r=$ 
0.07. From the above numerical examples, the method proposed in this paper provides the best result among three methods for analysis and synthesis problems of $\mathrm{H} \infty$ control.

\section{Conclusion}

New equivalent LMI representations to BRL have been derived for linear continuous-time systems. By introducing a new matrix variable, although some computation complexity has been increased, the new representation proposed here provides us with the possibility to obtain better results than previous methods. It improves the results that have been obtained before not only for $\mathrm{H} \infty$ norm computation but also state-feedback design of linear continuous-time systems with polytopic-type uncertainty. We can conjecture that this approach may be useful for extension to other control performance synthesis problem of these systems.

\section{Acknowledgments}

This work is supported by the National Natural Science Foundation of China (Grant no. 60704022) and Guangdong Natural Science Foundation (Grant no. 07006470).

\section{References}

[1] S. Boyd, L. EI Ghaoui, E. Feron, and V. Balakrishnan, Linear Matrix Inequalities in System and Control Theory, vol. 15 of SIAM Studies in Applied Mathematics, SIAM, Philadelphia, Pa, USA, 1994.

[2] G. Beck, A. Packard, D. Philbrick, and G. Blas, "Control of parameterically-dependent linear systems: a single quadratic Lyapunov approach," in Proceedings of the American Control Conference (ACC '93), pp. 2795-2799, San Francisco, Calif, USA, January 1993.

[3] R. M. Palhares, R. H. C. Takahashi, and P. L. D. Peres, "H $\infty$ and H2 guaranteed costs computation for uncertain linear systems," International Journal of Systems Science, vol. 28, no. 2, pp. 183-188, 1997.

[4] E. Feron, P. Apkarian, and P. Gahinet, "Analysis and synthesis of robust control systems via parameter-dependent Lyapunov functions," IEEE Transactions on Automatic Control, vol. 41, no. 7, pp. 1041-1046, 1996.

[5] P. Gahinet, P. Apkarian, and M. Chilali, "Affine parameter-dependent Lyapunov functions and real parametric uncertainty," IEEE Transactions on Automatic Control, vol. 41, no. 3, pp. 436-442, 1996.

[6] P. Apkarian and H. D. Tuan, "Parameterized LMIs in control theory," SIAM Journal on Control and Optimization, vol. 38, no. 4, pp. 1241-1264, 2000.

[7] J. C. Geromel, M. C. de Oliveira, and L. Hsu, "LMI characterization of structure and robust stability," Linear Algebra and Its Applications, vol. 285, no. 1-3, pp. 69-80, 1998.

[8] W. Xie, "Quadratic L2 gain performance LPV system design by a LTI controller with ILMI algorithm," IEE Proceedings-Control Theory and Applications, vol. 152, no. 2, pp. 125-128, 2005.

[9] M. C. de Oliveira, J. C. Geromel, and J. Bernussou, "Extended H2 and Ho norm characterizations and controller parametrizations for discrete-time systems," International Journal of Control, vol. 75, no. 9, pp. 666-679, 2002.

[10] Y. Ebihara and T. Hagiwara, "New dilated LMI characterizations for continuous-time multiobjective controller synthesis," Automatica, vol. 40, no. 11, pp. 2003-2009, 2004.

[11] U. Shaked, "Improved LMI representations for the analysis and the design of continuous-time systems with polytopic type uncertainty," IEEE Transactions on Automatic Control, vol. 46, no. 4, pp. 652-656, 2001.

[12] Y. Jia, "Alternative proofs for improved LMI representations for the analysis and the design of continuous-time systems with polytopic type uncertainty: a predictive approach," IEEE Transactions on Automatic Control, vol. 48, no. 8, pp. 1413-1416, 2003.

[13] P. J. de Oliveira, R. C. L. F. Oliveira, V. J. S. Leite, V. F. Montagner, and P. L. D. Peres, "Hoo guaranteed cost computation by means of parameter-dependent Lyapunov functions," Automatica, vol. 40, no. 6, pp. 1053-1061, 2004.

[14] P. Gahinet, A. Nemirovski, A. J. Laub, and M. Chilali, LMI control toolbox for use with matlab, Use's guide, The Math Works, Natick, Mass, USA, 1995. 